\title{
Salvage Transplantation with Cord Blood for Graft Rejection of Peripheral Blood Stem Cells due to Donor Specific Antibody
}

\author{
Maria Regina Pelobello de Leon ${ }^{1,2}$, Shuichiro Takahashi ${ }^{1,3}$, Masahiro Onozawa ${ }^{1}$, Makoto Ito ${ }^{4}$, Manabu Nakano ${ }^{5}$, Hajime \\ Senjo', Masahiro Chiba' ${ }^{1}$, Hiroyuki Ohigashi ${ }^{1}$, Emi Yokoyama ${ }^{1}$, Junichi Sugita ${ }^{1}$, Daigo Hashimoto ${ }^{1}$, Takanori Teshima ${ }^{1}$ \\ ${ }^{1}$ Department of Hematology, Faculty of Medicine, Hokkaido University, Sapporo, Japan, ${ }^{2}$ Department of Medicine, \\ Section of Hematology, Makati Medical Center, Makati City, Phillippines, ${ }^{3}$ Sapporo Hokuyu Hospital, Sapporo, Japan, \\ ${ }^{4}$ Division of Laboratory and Transfusion Medicine, Hokkaido University Hospital, Sapporo, Japan, ${ }^{5}$ Japanese Red Cross \\ Hokkaido Block Blood Center, Sapporo, Japan
}

\section{Abstract}

Allogeneic hematopoietic stem cell transplantation (HSCT) is a curative therapy for various kinds of hematological malignancies and disorders. Recently, HLA-haploidentical stem cell transplantation with post-transplantation cyclophosphamide (PTCy-haplo HSCT) has been widely performed due to its safety and favorable immune recovery. However, graft rejection remains an obstacle to PTCy-haplo HSCT. Donor specific antibody (DSA) is considered to be a major factor of graft rejection of haplo HSCT. We herein present a case of graft rejection after PTCy haplo-HSCT due to DSA induced by pretransplant platelet transfusion after donor selection. The patient was dependent on platelet transfusion and had not received cytotoxic chemotherapy because he was diagnosed as myelodysplastic syndrome/myeloproliferative neoplasm-unclassifiable. We retrospectively confirmed the level of DSA just before the first transplantation and found that it was dramatically elevated, which was enough to cause graft rejection. We successfully performed cord blood transplantation of the HLA that was not the target of DSA, as salvage transplantation without any desensitization. This case illustrates that we have to confirm the presence of DSA immediately before the haplo-HSCT, particularly in high risk patients who are dependent on platelet transfusion and have no cytotoxic chemotherapy before HSCT.

Key words: haploidentical transplantation, graft rejection, donor specific antibody, posttransplantation cyclophosphamide, cord blood transplantation

Submitted May 18, 2020; Accepted June 26, 2020; Published online August 31, 2020; Issued online November 25, 2020

Correspondence: Shuichiro Takahashi, Department of Hematology, Sapporo Hokuyu Hospital, Sapporo, Japan, 6-6-5-1, Higasi-Sapporo, Shiroishi-ku, Sapporo, Japan, 003-0006, E-mail: s_takahashi@ hokuyu-aoth.org

\section{Introduction}

Allogeneic hematopoietic stem cell transplantation (HSCT) is a curative therapy for various hematologic disorders. While the use of HLA-matched donors remains the standard of care, availability of these donors is limited. HLA-haploidentical HSCT using post-transplantation cyclophosphamide (PTCy) has been increasingly performed due to its low non-relapse mortality and favorable immune recovery ${ }^{1}$. However, graft rejection remains a major problem in haploidentical HSCT. It occurs in approximately $5-15 \%$ of patients and the presence of donor specific antibodies (DSA) is a risk factor for graft failure $^{2,3}$. DSA is detected in 10-21\% of haploidentical HSCT recipients ${ }^{4,5}$. While anti-HLA antibodies may develop spontaneously, they usually develop after exposure to non-self HLA molecules, such as during previous pregnancies, transfusions, or transplantations. Screening for DSA is recommended in all patients undergoing haploidentical HSCT. However, there is no recommendation regarding the timing and frequency of testing. We present a case of graft rejection after PTCy haplo-peripheral blood stem cell transplantation (PBSCT) due to DSA induced by pretransplant platelet transfusion after donor selection. 
Table 1. HLA typings of recipient and donors

\begin{tabular}{|c|c|c|c|c|c|c|c|c|}
\hline \multirow[b]{2}{*}{ Recipient } & \multicolumn{2}{|c|}{ HLA-A } & \multicolumn{2}{|c|}{ HLA-B } & \multicolumn{2}{|c|}{ HLA-C } & \multicolumn{2}{|c|}{ HLA-DR } \\
\hline & 24: 02 & 24: 02 & 52: 01 & 52: 01 & 12: 02 & 12: 02 & 09: 01 & 13: 02 \\
\hline Haplo donor & 24: 02 & 26: 02 & 48: 01 & 52: 01 & 08: 01 & 12: 02 & 09: 01 & 13: 02 \\
\hline Cord blood & 24: 02 & 24: 02 & 52: 01 & 52: 01 & 12: 02 & 12: 02 & 15: 02 & 15: 02 \\
\hline
\end{tabular}

\section{Case presentation}

A 58-year-old male was diagnosed with myelodysplastic syndrome/myeloproliferative neoplasm-unclassifiable (IPSS-R score 4), with trisomy 8 and associated enterocolitis. He was administered azacitidine (AZA) and referred to our institute for HSCT. Due to the lack of an available matched related or suitable unrelated donor, he was scheduled for a haplo-PBSCT with his son as the donor. Anti-HLA antibody screening carried out after the second cycle of AZA ( 83 days prior to transplantation) was positive for antibodies against several donor MHC class I antigens, but their mean fluorescence intensity was $<500$, which was the level of significance for DSA. He received 18 platelet transfusions between the second and fourth cycles of AZA prior to PBSCT.

He received related haplo-PBSCT (three locus mismatch for host-versus graft [HVG] direction; full match for graft-versus-host direction [Table 1]; CD34 ${ }^{+}$cell count of $6.91 \times 10^{6} / \mathrm{kg}$ ), following reduced-intensity conditioning consisting of fludarabine, busulfan, and 4 Gy total body irradiation. GVHD prophylaxis consisted of PTCy on days 3 and 4, followed by tacrolimus and mycophenolate mofetil from day 5. No haplo-immune fever developed. Neutrophil engraftment was not observed two weeks after HSCT. Bone marrow aspiration on day 20 after HSCT showed hypocellular marrow, with $100 \%$ recipient chimerism by short-tandem-repeat assay, and a diagnosis of graft rejection was made. A repeat screening for anti-HLA antibodies on day 23 showed strong positivity against a large number of antigens, including DSA to HLA-A26: 02 and HLA-B48: 01 (mean fluorescence intensity [MFI] levels; HLA-A26: 02 16548, HLA-B48: 01 2350). We retrospectively investigated the levels of DSA to HLA-A26: 02 and B48: 01 in the patient's serum harvested 7 days before the first HSCT. We found that both DSAs had already developed immediately before HSCT (MFI levels; HLA-A26: 02 21978, HLA-B48: 01 5228). Additionally, we checked the HLA typing of platelet transfusion donors to evaluate whether he had been sensitized and produced DSA by platelet transfusion. The results of HLA typing of platelets transfused between donor selection and first HSCT indicated that he received platelet transfusion, with HLA-A26 antigen twice with 4 weeks interval and HLA-B48 antigen once before HSCT (Figure 1). We selected the umbilical cord blood, which did not have any of the antigens tar- geted by the recipient's anti-HLA antibodies, as a salvage graft source (Table 1). He was administered a conditioning regimen comprising fludarabine, melphalan, and TBI 4 Gy, followed by cord blood transplantation (CBT, NCC $1.97 \times 10^{7} / \mathrm{kg}, \mathrm{CD} 34^{+}$cells $1.13 \times 10^{5} / \mathrm{kg}$, one locus mismatch for HVG and two locus mismatch for GVH direction; Table 1) on day 28 after the first HSCT. GVHD prophylaxis consisted of tacrolimus and short course methotrexate. He achieved neutrophil engraftment on day 18 after CBT. Bone marrow study on day 32 after CBT revealed a normocellular marrow with complete donor chimerism and $0.8 \%+8$ FISH positive cells. He was discharged on day 60 after the second HSCT.

\section{Discussion}

Haplo-HSCT using PTCy as a GVHD prophylaxis has been broadly accepted owing to its low incidence of acute and chronic GVHD and non-relapse mortality ${ }^{6}$. In a previous study, GVHD prophylaxis with PTCy showed excellent outcomes for PBSCT from an HLA-matched unrelated donor as well ${ }^{7}$. In this case, although there was no mismatch in the GVH direction, we considered GVHD prophylaxis with PTCy would be best for this patient due to its low incidence of GVHD and nonrelapse mortality.

In animal models, preexisting DSA can cause graft rejection within a few hours of HSCT $^{5}$. In humans, a high level of pre-transplant DSA directed against HLA class I antigens can cause hyper-acute rejection and immediate graft loss, while DSA against HLA class II antigens can cause rejection 2-4 days after transplant ${ }^{8}$. Haploimmune fever may develop due to the proliferation of alloreactive donor $\mathrm{T}$ cells exposed to host antigens. Although it develops in $96 \%$ of patients on days 3 and 4 following haplo-PBSCT using our protocol ${ }^{9}$, it did not develop in our patient, suggesting that graft rejection had occurred at that time. This patient had no HLA mismatch in the GVH direction despite three mismatches in the HVG direction, and we did not use any immunosuppressants before the day of PTCy. These conditions and the high levels of DSA might have resulted in host immune cells activating and causing graft rejection.

According to a previous report, six or more blood transfusions before haplo-HSCT is a risk factor for the production of HLA antibodies ${ }^{4}$. MDS patients are often dependent on transfusion before HSCT. Thus, MDS 


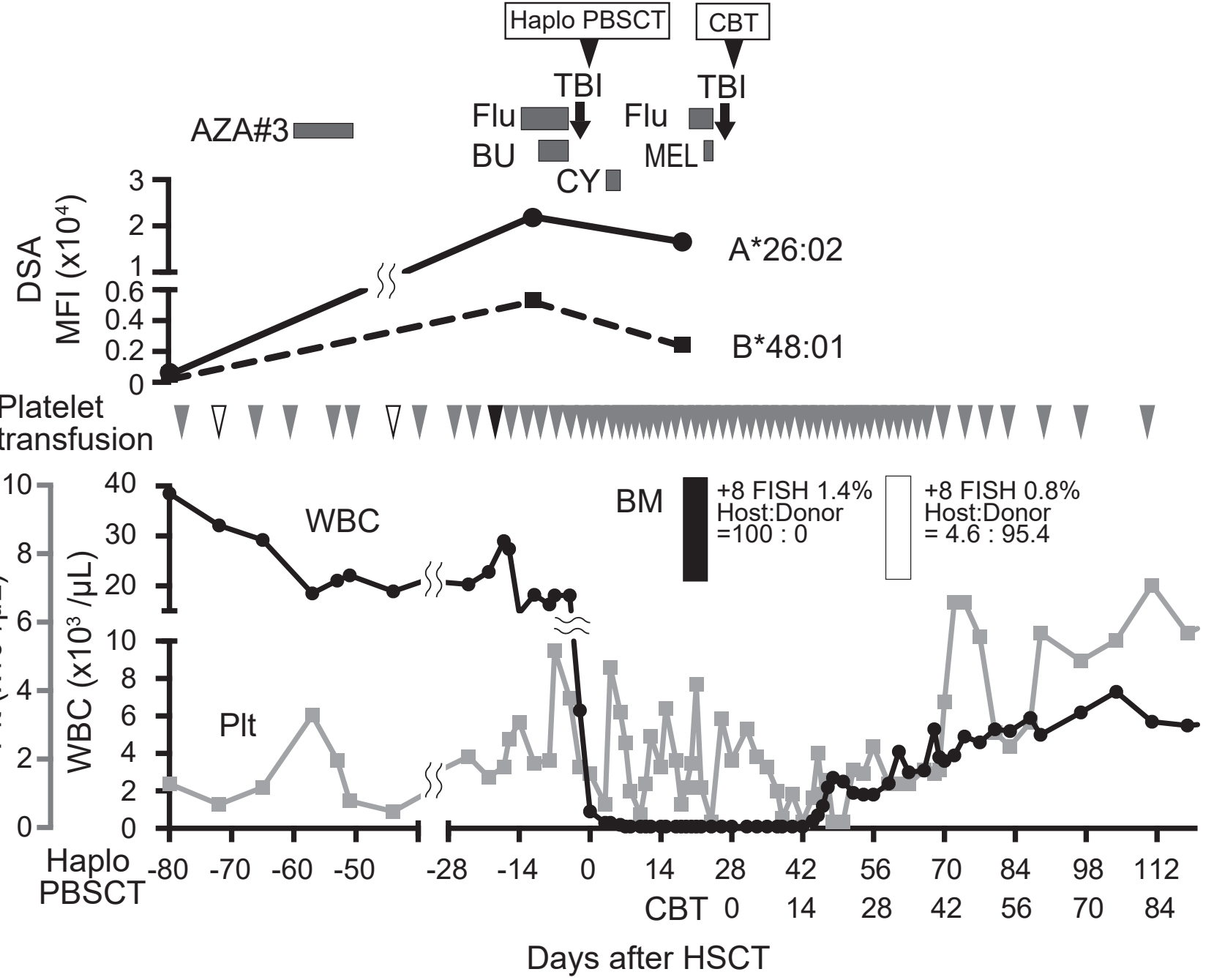

Figure 1. Clinical time course and the levels of DSA

No significant DSA was observed at donor selection prior to Haplo-PBSCT with an increased level of DSA at the time of re-assessment 7 days before and 23 days after HSCT. White triangles show the platelets having HLA-A26 antigen, and the black triangle shows the platelet having HLA-B48 antigen.

AZA, azacitidine; BU, busulfan; CY, cyclophosphamaide; Flu, fludarabine; TBI, total body irradiation; DSA, donor specific antibody.

patients should be considered to be at a high risk for the generation of HLA antibodies. In this case, platelet transfusions induced enough production of DSA to cause graft rejection. Although a recent report showed successful rescue transplantation by another DSA targeted haploidentical donor using desensitization procedure ${ }^{10}$, successful desensitization is not always guaranteed even if multiple methods are combined including plasma exchange, rituximab, bortezomib, IVIg, and/or DSA-PC as a bait. We chose HLA class I identical cord blood for the second transplantation, which theoretically would never be targeted by DSA targeting HLA class I, and the patient achieved successful engraftment.

In conclusion, since the development of anti-HLA antibodies is drastic and has a significant impact on engraftment even after PBSCT, re-assessment of DSA should be considered for recipients who needed several platelet transfusions without cytotoxic chemotherapy or immunosuppressive therapy after donor selection.

\section{Author Contributions}

M. R. P. L. and S. T. wrote this manuscript. M. O., J. S., D. H. and T. T. revised this manuscript. M. I. and M. N. performed HLA typing and the analysis of DSA. H. S., M. C., H. O. and E. Y. collected the data. All authors read and approved the final manuscript.

\section{Conflicts of Interest}

The authors declare no conflicts of interest associated with this article. Disclosure forms provided by the authors are available here. 


\section{References}

1. Kanakry CG, Fuchs EJ, Luznik L. Modern approaches to HLA-haploidentical blood or marrow transplantation. Nat Rev Clin Oncol. 2016; 13: 10-24.

2. Sugita J. HLA-haploidentical stem cell transplantation using posttransplant cyclophosphamide. Int J Hematol. 2019; 110: 30-8.

3. Ciurea SO, de Lima M, Cano P, Korbling M, Giralt S, Shpall EJ, et al. High risk of graft failure in patients with anti-HLA antibodies undergoing haploidentical stem-cell transplantation. Transplantation. 2009; 88: 1019-24.

4. Huo MR, Xu YJ, Zhai SZ, Lv M, Wang Y, Cao LQ, et al. Prevalence and risk factors of antibodies to human leukocyte antigens in haploidentical stem cell transplantation candidates: A multi-center study. Hum Immunol. 2018; 79: 672-7.

5. Ciurea SO, Cao K, Fernandez-Vina M, Kongtim P, Malki MA, Fuchs E, et al. The European Society for Blood and Marrow Transplantation (EBMT) Consensus Guidelines for the Detection and Treatment of Donor-specific Anti-HLA Antibodies (DSA) in Haploidentical Hematopoietic Cell Transplantation. Bone Marrow Transplant. 2018; 53: 521-34.

6. Luznik L, O’Donnell PV, Symons HJ, Chen AR, Leffell MS, Zahurak M, et al. HLA-haploidentical bone marrow transplantation for hematologic malignancies using nonmyeloablative conditioning and high-dose, posttransplantation cyclophospha- mide. Biol Blood Marrow Transplant. 2008; 14: 641-50.

7. Bolanos-Meade J, Reshef R, Fraser R, Fei M, Abhyankar S, Al-Kadhimi Z, et al. Three prophylaxis regimens (tacrolimus, mycophenolate mofetil, and cyclophosphamide; tacrolimus, methotrexate, and bortezomib; or tacrolimus, methotrexate, and maraviroc) versus tacrolimus and methotrexate for prevention of graft-versus-host disease with haemopoietic cell transplantation with reduced-intensity conditioning: a randomised phase 2 trial with a non-randomised contemporaneous control group (BMT CTN 1203). Lancet Haematol. 2019; 6: e132-43.

8. Hickey MJ, Valenzuela NM, Reed EF. Alloantibody Generation and Effector Function Following Sensitization to Human Leukocyte Antigen. Front Immunol. 2016; 7: 30.

9. Sugita J, Kagaya Y, Miyamoto T, Shibasaki Y, Nagafuji K, Ota $\mathrm{S}$, et al. Myeloablative and reduced-intensity conditioning in HLA-haploidentical peripheral blood stem cell transplantation using post-transplant cyclophosphamide. Bone Marrow Transplant. 2019; 54: 432-41.

10. Minami M, Matsushima T, Mori Y, Ishihara D, Jinnnouchi F, Takenaka K, et al. Successful rescue transplantation with desensitization procedure after primary graft failure due to donor-specific antibody. Bone Marrow Transplant. 2019; 54: 1374-6.

https://doi.org/10.31547/bct-2020-004

Copyright (C) 2020 APBMT. All Rights Reserved. 